\title{
A method to prevent cerebrospinal fluid leakage: Reinforcing acellular dermal matrix
}

\author{
Hojune Lee ${ }^{1 *}$, \\ Ye Seul Eom ${ }^{2 *}$, \\ Jai-Kyong Pyon ${ }^{1}$ \\ ${ }^{1}$ Department of Plastic Surgery, \\ Samsung Medical Center, \\ Sungkyunkwan University School of \\ Medicine, Seoul; ${ }^{2} J J$ Plastic Surgery \\ Center, Seoul, Korea
}

This article contains Supplemental Video S1.

\begin{abstract}
In neurosurgical cases, problems related to wound healing can vary from simple wound dehiscence to multilayer defects. This study demonstrates an effective method to prevent persistent cerebrospinal fluid (CSF) leakage using reinforcing acellular dermal matrix in neurosurgical patients with wound dehiscence. A 52-year-old woman was admitted for management of recurrent glioblastoma. After tumor removal surgery, the patient experienced sustained CSF leakage from the wound despite reparative attempts. The plastic surgery team performed wound repair procedure after remnant tumor removal by the neurosurgery team. Acellular dermal matrix was applied over the mesh plate to prevent CSF leakage and the postoperative status of the patient was evaluated. No sign of CSF leakage was found in the immediate postoperative period. After 3 years, there were no complications including CSF leakage, wound dehiscence, and infection. We hereby propose this method as a feasible therapeutic alternative for preventing CSF leakage in patients experiencing wound problem after neurosurgical procedures.
\end{abstract}

Keywords: Acellular dermis / Cerebrospinal fluid leakage / Surgical wound dehiscence

\section{INTRODUCTION}

In neurosurgical cases, complications in wound healing vary from simple wound dehiscence to complex multilayer defects, which can result in cerebrospinal fluid leakage and contamination [1]. Risk factors such as irradiation, multiple previous attempts at wound closure, and infections may be linked and lead to a vicious cycle of complications and treatment. Among these wound-related complications, cerebrospinal fluid leakage is considered one of the most serious, particularly in neurosurgical field $[1,2]$. To prevent these complications, several surgical approaches such as skin grafting, loco-regional/micro-vascular flaps, and nonsurgical approaches using liquid skin sealants

Correspondence: Jai-Kyong Pyon

Department of Plastic Surgery, Samsung Medical Center, Sungkyunkwan

University School of Medicine, 81 Irwon-ro, Gangnam-gu, Seoul 06351, Korea

E-mail: pspriest.pyon@samsung.com

*The first two authors contributed equally to this work.

Received October 1, 2019 / Revised January 9, 2020 / Accepted January 23, 2020 have been introduced [3].

In the past few years, acellular dermal matrix has been used for reconstruction following burns or complex wounds of the scalp [4]. Furthermore, several studies have demonstrated the effectiveness of acellular dermal matrix reinforcement in prevention/treatment of cerebrospinal fluid leakage [5-8]. However, the studies lack representative case reports and detailed descriptions of the surgical technique. In this report, we describe details of a case and outcome of an effective coverage method with acellular dermal matrix in a patient who experienced wound dehiscence due to cerebrospinal fluid leakage.

\section{CASE REPORT}

A 52-year-old woman presented to the emergency room with clear discharge from her surgical wound. Medical history revealed that the patient had undergone surgery thrice for removal of brain tumor along with 30 sessions of cranial radio- 
therapy. The last surgery was performed 2 months before. During the procedure, the recurring tumor was successfully removed, and primary closure of the dura was performed using continuous locking sutures. One month following the surgery, the patient was discharged without any complications. However, on postoperative day 62 , the patient visited our emergency room with discharge of fluid from the surgical wound and nose that appeared to be cerebrospinal fluid. The patient was admitted to the neurosurgery department, where a team added several sutures to the previous incision line and applied compressive dressing. Despite these procedures, the leakage persisted (Fig. 1A). Furthermore, brain computed tomography imaging revealed tumor progression from the right parietotemporal area, which was the site of previous tumorectomy, and extradural fluid collection in the cavity with leakage site on the posterior side of the wound (Fig. 1B). For these reasons, the neurosur-
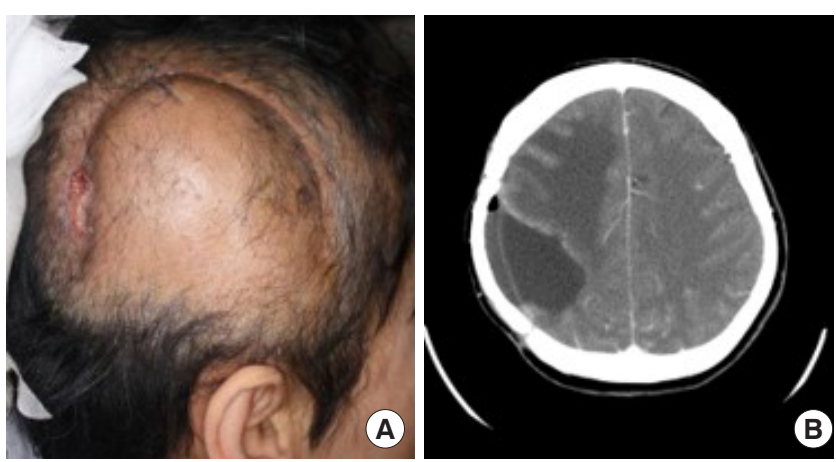

Fig. 1. Various methods used to prevent cerebrospinal fluid leakage. (A) Additional sutures applied at the incision site were unsuccessful in stopping the leakage. (B) Preoperative brain computed tomography imaging of the patient, which presents extradural fluid collection in the right parietotemporal area. gery team decided to insert a lumbar drain and consulted with our plastic surgery team regarding the wound-related complication.

We first attempted negative pressure wound therapy, which promotes vessel destabilization and maturation. CuraVAC (Daewoong Pharm Co., Ltd., Seoul, Korea) was fixed to the shaved scalp using 3M Ioban 2 (3M Health Care Inc., St. Paul, MN, USA). One month following negative pressure wound therapy, persistent wound dehiscence was observed. We performed selective debridement and applied Dermabond on the incision line.

Due to non-improvement in the condition, the neurosurgery team decided to perform repeat surgery for remnant tumor removal and wound repair. In the operating room, following the previous operative incision, the neurosurgery team excised the remnant tumor with necrotic tissue and performed duroplasty with fascia lata. After duroplasty, a mesh plate was inserted as a calvarial substitute. The plastic surgery team joined the operation and applied a $4 \times 16 \mathrm{~cm}$ section of CGCryoDerm (CGBio Co., Seongnam, Korea) over the mesh plate, especially beneath the incision site to prevent cerebrospinal fluid leakage. A drain was inserted and primary closure was performed (Fig. 2). Negative pressure wound therapy was applied for a week (Supplementary Video S1).

On postoperative day 2 , the color of the drainage changed from serosanguineous into serous drainage which became nearly close to that of the cerebrospinal fluid. Under the judgement of neurosurgical experts, the drain was removed not to disrupt the cerebrospinal fluid circulation system. On postoperative day 8 , the negative pressure wound therapy was stopped and no sign of leakage was observed. At the 5-month and
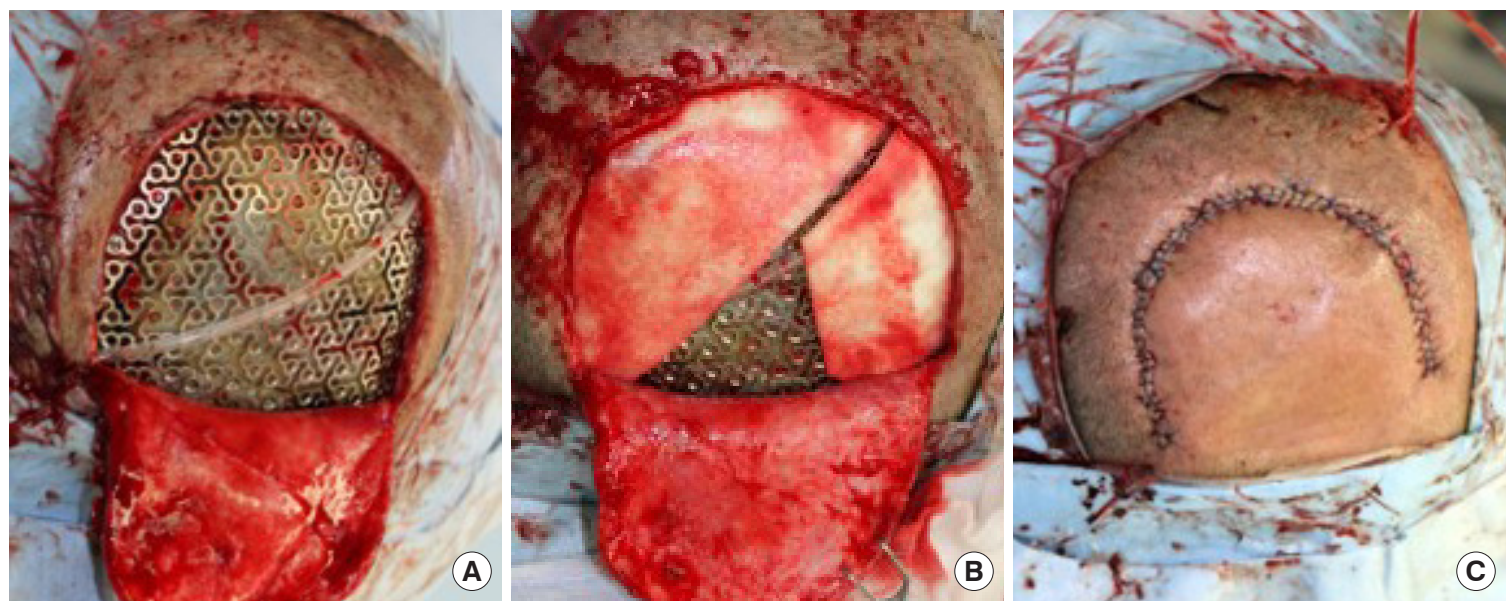

Fig. 2. Surgical process of acellular dermal matrix augmentation. (A) Neurosurgery team excised the remnant tumor followed by duroplasty and mesh plate insertion. (B) Plastic surgery team applied a section of acellular dermal matrix over the mesh plate, especially beneath the incision site. (C) A drain was inserted and primary closure was performed. 

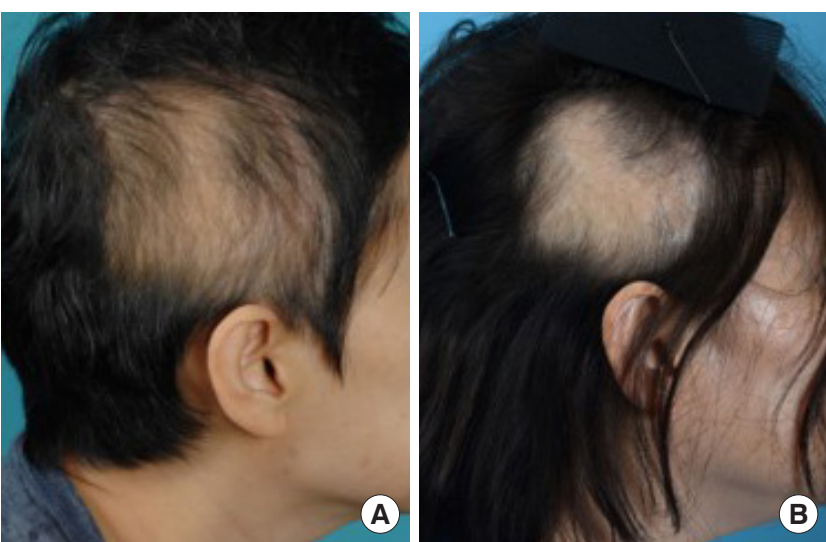

Fig. 3. Photographs after the final operation. (A) Photograph taken at the 5-month postoperative follow-up. (B) Photograph taken at the 3-year postoperative follow-up. No complications were found in patient including cerebrospinal fluid leakage and wound dehiscence.

3-year postoperative follow-up, there were no complications including cerebrospinal fluid leakage, wound dehiscence, or infection (Fig. 3).

\section{DISCUSSION}

Cerebrospinal fluid leakage is a common complication after neurosurgical procedures. Cerebrospinal fluid leakage and other factors such as cortisone therapy, irradiation, infection, and repeated (three times or more) attempts at wound closure have been associated with wound-related complications [1]. However, the mechanism of delayed wound healing due to cerebrospinal fluid leakage remains under debate, and some studies have reported that cerebrospinal fluid is more watery than serum; therefore, can result in maceration at the wound edges [2]. In the present case, the patient had cerebrospinal fluid leakage through the bony defect along with several risk factors for wound dehiscence. Different procedures were performed to prevent cerebrospinal fluid leakage including application of additional sutures, skin sealants, lumbar drain, and negative pressure wound therapy, which promotes vessel destabilization and maturation. However, the leakage persisted and collection of fluid was evident. Initially, we considered loco-regional or free flaps for the repair procedure. However, considering the high risk of relapse and possibility of delayed diagnosis of recurrence due to bulky flap design, we decided to apply acellular dermal matrix over the mesh plate.

The advantage of reinforcing acellular dermal matrix over the mesh plate is that it can potentially eliminate flap donor-site morbidity and complications. In addition, this acellular dermal matrix can be customized to any defect size, and is readily avail-

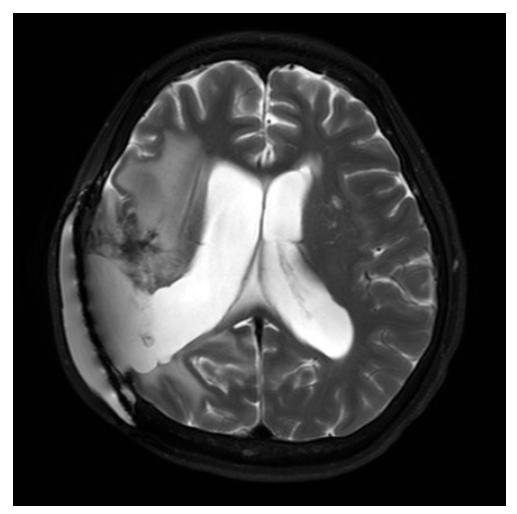

Fig. 4. Five-month postoperative brain magnetic resonance imaging (MRI) of the patient. MRI T2 weighted image shows cerebrospinal fluid accumulation still exists over the mesh plate (black line with patterned protuberance). Integrated acellular dermal matrix (black thin line) is found right above the outer cerebrospinal fluid accumulation which acts as an outer barrier.

able for use.

Acellular dermal matrix has been used in various fields. Few studies have demonstrated the effectiveness of acellular dermal matrix reinforcement in prevention of cerebrospinal fluid leakage [5-8]. Although use of acellular dermal matrix after neurosurgery is not a novel procedure, previous studies lack representative case reports including photographs and detailed descriptions of the surgical technique. Therefore, in this report, we described a typical case of wound-related complication with cerebrospinal fluid leakage, which was successfully treated by acellular dermal matrix reinforcement.

In previous studies [9] the fact that host response to acellular dermal matrix paralleling the normal wound healing has been introduced, indicating integration of acellular dermal matrix with scalp may done in only a few weeks. Five-month postoperative brain magnetic resonance imaging T2 weighted image shows integrated acellular dermal matrix as black thin line located right above the outer cerebrospinal fluid accumulation, which acts as a barrier (Fig. 4). Meanwhile, cerebrospinal fluid accumulation over the mesh plate (black line with patterned protuberance) indicates failure of capturing the cerebrospinal fluid under dura mater despite after the duroplasty. This supports the importance of reinforcement with acellular dermal matrix for prevention of cerebrospinal fluid leakage.

While several variants of acellular dermal matrix are commercially available, we used CGCryoDerm that has more preserved dermal structure owing to the manufacturing process, which involves freezing without drying. CGCryoDerm also contains abundant growth factors for angiogenesis and recellularization. Acellular dermal matrix is beneficial in reinforcing the native dermis, which can act as a double barrier to cerebrospinal fluid 
leakage. However, cautious postoperative evaluation is necessary since acellular dermal matrix may increase the risk of development of infection.

Although the patient in the present study had associated risk factors that included many prior sessions of radiation therapy, along with more than three wound repair procedures, she was free of complications including cerebrospinal fluid leakage and wound dehiscence at 3 years, postoperatively. This case illustrates that reinforcement with acellular dermal matrix can be a surgical option for preventing cerebrospinal fluid leakage even in problematic wounds.

\section{NOTES}

\section{Conflict of interest}

No potential conflict of interest relevant to this article was reported.

\section{Ethical approval}

The study was approved by the Institutional Review Board of Samsung Medical Center (IRB No. 2019-09-020-001) and performed in accordance with the principles of the Declaration of Helsinki. Written informed consent was obtained.

\section{Patient consent}

The patient provided written informed consent for the publication and the use of her images.

\section{ORCID}

Hojune Lee https://orcid.org/0000-0001-5226-1825

Ye Seul Eom https://orcid.org/0000-0002-3202-5873

Jai-Kyong Pyon https://orcid.org/0000-0002-6302-3002

\section{Supplementary material}

Supplementary Video S1. Acellular dermal matrix was applied over the mesh plate, especially beneath the incision site to prevent cerebrospinal fluid leakage. A drain was inserted and primary closure was performed followed by vacuum-assisted clo- sure dressing.

Supplemental data can be found at: https://doi.org/10.7181/ acfs.2019.00535.v001

\section{REFERENCES}

1. Krishnan KG, Muller A, Hong B, Potapov AA, Schackert G, Seifert V, et al. Complex wound-healing problems in neurosurgical patients: risk factors, grading and treatment strategy. Acta Neurochir (Wien) 2012;154:541-54.

2. Babuccu O, Kalayci M, Peksoy I, Kargi E, Cagavi F, Numanoglu G. Effect of cerebrospinal fluid leakage on wound healing in flap surgery: histological evaluation. Pediatr Neurosurg 2004; 40:101-6.

3. Rotenberg BW, Marchie A, Cusimano MD. Skin sealants: an effective option for closing cerebrospinal fluid leakage. Can J Surg 2004;47:466-8.

4. Brunetti B, Tenna S, Segreto F, Persichetti P. The use of acellular dermal matrix in reconstruction of complex scalp defects. Dermatol Surg 2011;37:527-9.

5. Prickett KK, Wise SK, Delgaudio JM. Choice of graft material and postoperative healing in endoscopic repair of cerebrospinal fluid leak. Arch Otolaryngol Head Neck Surg 2011;137: 457-61.

6. Ismail AS, Costantino PD, Sen C. Transnasal transsphenoidal endoscopic repair of CSF leakage using multilayer acellular dermis. Skull Base 2007;17:125-32.

7. Bowers CA, Brimley C, Cole C, Gluf W, Schmidt RH. AlloDerm for duraplasty in Chiari malformation: superior outcomes. Acta Neurochir (Wien) 2015;157:507-11.

8. Mericli AF, Gampper TJ. The use of an acellular dermal matrix "sandwich" for the successful treatment of a persistent cranial cerebrospinal fluid fistula. J Craniofac Surg 2013;24:1314-6.

9. Wong AK, Schonmeyr B, Singh P, Carlson DL, Li S, Mehrara BJ. Histologic analysis of angiogenesis and lymphangiogenesis in acellular human dermis. Plast Reconstr Surg 2008;121:114452. 